\title{
Threat of Exit as a Source of Bargaining Power
}

\author{
Fabian BERGÈS \\ Toulouse School of Economics (GREMAQ-INRA, IDEI) \\ and \\ Claire CHAMBOLLE \\ INRA (Department of Economics at École Polytechnique)
}

\section{Corresponding author :}

Fabian BERGÈS

Manufacture des Tabacs - MF 318

21 Allée de Brienne

$\mathrm{F}-31000$ Toulouse

(France)

Phone : +33561128580
Fax : +33561225563

Mail : Fabian.Berges@toulouse.inra.fr

\section{Second author :}
Claire CHAMBOLLE
1 rue Descartes
F - 75005 Paris
(France)

Phone: +33146343215

Fax: +33146343428

Mail : chamboll@ivry.inra.fr 


\title{
Threat of Exit as a Source of Bargaining Power*
}

\begin{abstract}
This article analyzes a simple two-period model where two homogenous manufacturers compete to supply a monopolist retailer. We show that, if manufacturers are vulnerable, i.e if they are likely to exit the market in case of insufficient orders in the first period, they may exploit their threat of exit to capture the whole first period industry profit. Indeed, the retailer will accept to pay the high price to the manufacturers in order to secure upstream competition in the second period. Results are robust under different market structures or contract types.
\end{abstract}

JEL Classifications: L14, L12, D21, Q12.

Keywords: Bargaining power, market entry, vertical contract.

${ }^{*}$ We thank Roman Inderst, Patrick Rey, Thibaud Vergé, and seminars participants at EARIE 2005 and EEA 2006. The title of a previous version of this article was: 'The Reciprocal Producer's Incentives to Prey and Retailer's Buyer Power' 


\section{Introduction}

The industrial organization literature has recently devoted a great deal of interest to the analysis of the determinants of bargaining power in vertical relationships. Bargaining power deals with firms' capacity to capture the surplus created by a transaction within the vertical structure. Dobson \& Waterson (1999) and Allain (2002) show that the determinants of such a balance of power between producers and retailers seems to be inversely correlated with the relative degree of imperfect competition at the considered level. More competition downstream translates thus into more bargaining power upstream. Other sources of bargaining power have been recently highlighted, such as retailer's size associated with buyer power in Inderst and Wey (2007) or producer's differentiation in Chambolle and Villas-Boas (2007). For a recent and wider survey on the buyer power and its determinants, see Inderst and Mazzarotto (2006).

In this article, we show that the threat of exit may be used as a particular weapon in the negotiation between producers and retailers. This article reveals that producers who are highly dependent from a retailer (in the sense that a temporary breach in their contract with the retailer may credibly induce their exit out of the market) may turn this apparent weakness into a strategy resulting in an increase of their profits. A buyer may indeed accept higher wholesale prices from its suppliers in order to preserve future upstream competition, and thus future profits. We characterize pure strategy Nash equilibria where producers increase their bargaining power by neutralizing upstream competition. They are able to capture the whole surplus created by their transaction with the retailer, exploiting the buyer's dependance on future competition that may be jeopardized by the threat of manufacturers' exit, and increasing therefore their bargaining power. Financial constraints have a key role to explain why these equilibria are sustainable.

Related results can be found in the procurement literature devoted to single vs dual sourcing in buyer's strategy where a principal faces two agents for supply. In a learning by doing context 
where sellers' production cost decreases with the quantity produced at each period, Lewis and Yildirim (2002) exhibit equilibria where the buyer orders alternatively from different sellers to maintain future market rivalry rather than buying exclusively from one manufacturer at a lower cost. Biglaiser and Vettas (2004) develop a model based on a different assumption: a capacity constrained producer disappears once he sold its entire production. They however fail to find pure strategy equilibria, but they show that a buyer may split his orders to preserve future competition on the market. Repeated interactions between manufacturers and retailers may therefore change the classic one shot outcome where only the efficient manufacturer is active.

We first develop a simple model that confirms our main argument: a retailer may loose buyer power in a dynamic framework of repeated contract because of his will to maintain future upstream competition. We then relax some assumptions on contract types or market structure and show that the mechanism still hold, giving thus robustness to our result.

\section{The Model}

Two upstream manufacturers $M 1$ and $M 2$ compete for the production of a homogenous product. A downstream monopolist retailer $R$ carries the product and resells it to final consumers. Consumers demand at most two units of the good per period, and their willingness to pay per unit is normalized to 1. Each manufacturer can produce at most two units of the good per period. While $M 1$ has a zero unit cost, $M 2$ is assumed to be less efficient than $M 1$ with a unit $\operatorname{cost} c \in[0,1]$.

We add the three following assumptions:

A1: If no unit is bought to a manufacturer for one period, he exits the market at the end of this period.

A2: Manufacturers have a discount factor $\delta^{m}$ whereas the retailer has a discount factor $\delta^{r}$. Besides, $\delta^{m}<\delta^{r}$. 
A3: There is no entry on the upstream market.

Assumptions $A 1$ and $A 2$ translate manufacturers' structural and financial vulnerability. If a manufacturer receives an insufficient order in $t=1$, he won't be active in $t=2$ (sales objectives). ${ }^{1}$ We assume a lower discount factor for manufacturers relatively to the retailer since present profits are more important for a vulnerable firm. Besides, this assumption translates the fact that a bank will not offer the same credit conditions to a powerful retailer or to vulnerable manufacturers (risky investment). ${ }^{2}$

We consider a two-period game where $t=\{1,2\}$ denotes the period. The timing for each period is the following. At stage 1, M1 and M2 make simultaneously take-it or leave-it wholesale price offers to the retailer, respectively denoted $w_{1}^{t}$ and $w_{2}^{t} \cdot{ }^{3}$ At stage 2 , the retailer decides his supply strategy, i.e. he can either buy exclusively from $M 1$ or from $M 2$, or he can purchase one unit to each of them. At stage 3, the retailer sets the final price and resells the units bought to consumers.

In a one-shot game, the classic equilibrium is the Bertrand solution. Manufacturers offers are $w_{1}=c-\varepsilon, w_{2}=c$ and the retailer buys only from $M 1$. Profits are: $\Pi_{M 1}=2 c, \Pi_{M 2}=0$ and $\Pi_{R}=2(1-c)$. Price competition leads the efficient manufacturer to be the exclusive supplier with strictly positive profits because of his competitive advantage. The retailer also gets positive profits thanks to upstream competition and social welfare is maximal since the two units are produced by the most efficient manufacturer: $S W=2$.

\footnotetext{
${ }^{1}$ The firm can need to meet profit hurdles to remain on the market in the future, as argued in Biglaiser and DeGraba (2001) or Bolton and Scharfstein (1990).

${ }^{2}$ For a model of firms' financing through imperfect capital market, see Holström and Tirole (1997). They show why risky investments, in presence of moral hazard, are less likely to be financed for firms with low assets.

${ }^{3}$ We assume that producers and retailer are unable to commit to any long term contract because of the uncertain presence of manufacturers at the second period. Note that we also exclude all other types of transfer (transfers not based on sales) between the retailer and the manufacturers.
} 


\section{The Two-period Game Equilibria}

In a two-period game, the possibility for one manufacturer to exit the market at the end of period 1 if he didn't sell one unit to the retailer generates new equilibria. We solve the game backward to find the Nash subgame equilibria.

\subsection{Subgame equilibria in $t=2$}

There are three subgame equilibria depending on the supply strategy the retailer decided in $t=1$. If $R$ chose to buy 2 units to $M 1$ (resp. $M 2)$ in $t=1, M 2$ (resp. $M 1)$ is no more active in $t=2$. Therefore in $t=2, M 1$ (resp. $M 2)$ offers a monopolist contract $w_{1}^{2}=1\left(\right.$ resp. $\left.w_{2}^{2}=1\right)$ to $R$, who accepts. $R$ sets then the final price at $p^{2}=1$ and resells both units to consumers. Profits are thus: $\Pi_{M 1}^{2}=2 \delta^{m}$ (resp. $\Pi_{M 2}^{2}=2 \delta^{m}(1-c)$ ), while $R$ gets zero profit.

If $R$ chose to buy one unit to each manufacturer, both $M 1$ and $M 2$ are still on the market in $t=2$. Therefore, upstream competition in $t=2$ leads to the one-shot game equilibrium and profits are: $\Pi_{M 1}^{2}=2 \delta^{m} c, \Pi_{M 2}^{2}=0$ and $\Pi_{R}^{2}=2 \delta^{r}(1-c)$.

\subsection{Equilibria in $t=1$}

In $t=1$, each manufacturer has the choice between an "Exclusion" (E) wholesale price to get the exclusive supply towards the retailer, or an "Accommodation" (A) strategy with his rival. Superscripts on variables denotes the chosen strategy.

First, a manufacturer adopts an exclusion strategy if he sets a sufficiently low wholesale price to convince the retailer to choose him as an exclusive supplier, renouncing to the benefits of upstream competition in $t=2$. We determine, for each manufacturer, the highest wholesale price which given his rival's price enables him to get the supply exclusivity in $t=1$.

Lemma $1 M 1$ 's predation strategy for exclusivity is $w_{1}^{1 E}=\max \left\{w_{2}^{1}-2 \delta^{r}(1-c),-\delta^{m}\right\}$. M2's exclusion strategy is $w_{2}^{1 E}=\max \left\{w_{1}^{1}-2 \delta^{r}(1-c), c-\delta^{m}(1-c)\right\}$. 
In order to get the exclusivity, each manufacturer must offer a first period wholesale price low enough to compensate the retailer for the absence of upstream competition in the second period: the foregone retailer's profit is $\pi_{R}^{2}=2 \delta^{r}(1-c)$. Moreover, both manufacturers and the retailer have to realize non negative profits over the two periods: therefore $w_{1}^{1 E}>-\delta^{m}$ and $w_{2}^{1 E}>c-\delta^{m}(1-c)$.

A manufacturer who chooses an accommodation strategy can afford to set a higher price than his rival. Indeed, manufacturers know that the retailer is reluctant to renounce to the benefits of upstream competition in $t=2$. In other words, manufacturers anticipate the retailer will be strongly disposed to buy one unit to each of them, and they thus seek to exploit such behavior. We determine, for each manufacturer, the highest acceptable wholesale price given the rival offer to supply one unit in $t=1$ to the retailer.

Lemma $2 M 1$ 's accommodation strategy is $w_{1}^{1 A}=\max \left\{\min \left\{w_{2}^{1}+2 \delta^{r}(1-c), 2+2 \delta^{r}(1-c)-w_{2}^{1}\right\} ; 0\right\}$. M2's accommodation strategy is $w_{2}^{1 A}=\max \left\{\min \left\{w_{1}^{1}+2 \delta^{r}(1-c) ; 2+2 \delta^{r}(1-c)-w_{1}^{1}\right\} ; c\right\}$.

The retailer's financial constraint is taken into account by manufacturers who anticipate that the sum of their first-period wholesale prices must not exceed the total revenues the retailer then gets over the two periods: $w_{1}^{1 A}+w_{2}^{1 A} \leq 2+2 \delta^{r}(1-c)$. When the retailer's financial constraint is not binding, the accommodation wholesale price strictly increases with his rival price. Since each manufacturer anticipates that the retailer is ready to pay a premium to keep him active in the next period, it enables manufacturers to raise their price and thus relax upstream competition.

Let $\gamma$ be such that $\delta^{r}=\gamma \delta^{m}$. It results from $A 2$ that $\gamma>1$. The analysis of each manufacturer's incentive to deviate unilaterally from an "Accommodation" to a "Exclusion" strategy leads to the following proposition.

Proposition 1 For $\widetilde{\delta}=\frac{2-c}{2(1-c)(3 \gamma-2)}$ and $\delta^{*}=\frac{c}{2(1-c)(3 \gamma-1)}$, there exists two types of subgame perfect Nash equilibria in pure strategies : 
(i) If $\widetilde{\delta}<\delta^{m}<1$, there is a continuum of Nash equilibria accommodation defined by the following conditions: $w_{2}^{1 A} \in\left[\frac{1}{3}\left(4-c+2 \delta^{m}(1-c)\right), \frac{2}{3}\left(1+(3 \gamma-1) \delta^{m}(1-c)\right)\right]$ and $w_{1}^{1 A}+w_{2}^{1 A}=2+2 \delta^{r}(1-c)$. $R$ buys one unit to each supplier in $t=1$, and only to $M 1$ at price $w_{1}^{2}=c$ in $t=2$;

(ii) If $\delta^{*}<\delta^{m} \leq \widetilde{\delta}$, there is no Nash equilibrium in pure strategies.

(iii) If $0 \leq \delta^{m} \leq \delta^{*}$, the only Nash equilibrium (exclusivity) is such that $w_{1}^{1 E}=c-2 \delta^{r}(1-c)$, $w_{2}^{1 E}=c, R$ buys 2 units to $M 1$ (exclusivity) in $t=1$. $R$ then buys 2 units at price $w_{1}^{2 E}=1$ in $t=2$;

Proof. See Appendix.

The only exclusion equilibrium (iii) is such that $M 1$ gets the supply exclusivity since $M 2$ is less efficient. The predatory wholesale price for $M 1$ depends on the level of the premium he must pay to the retailer in comparison with $M 2$ 's marginal cost $c$. This premium increases in $\delta^{r}$ (and in $\delta^{m}$ ), since $R$ grants more value to the second period profits. On the contrary, the premium decreases with $c$ : When $M 2$ is less efficient, he is not a very interesting leverage for $R$ in $t=2$ (when $c$ increases, $R$ 's second period profit when both producers compete decreases). The exclusion equilibrium is thus sustainable for low values of the discount factors and the upper threshold $\delta^{*}$ increases with c.

For intermediate values of $\delta^{m}$ or $\delta^{r}$ (ii), as it becomes more costly for $M 1$ to prey his rival in $t=1, M 1$ has an incentive to switch for an accommodation strategy. However, the accommodation profits expected by $M 2$ in $t=1$ are not high enough and $M 2$ has now an incentive to exclude $M 1$ rather than to accommodate. Finally, there is no Nash equilibrium in pure strategies. 
When $\delta^{m}>\widetilde{\delta}(\mathrm{i})$, the expected accommodation profit is high while the premium to pay to get the exclusivity is too expensive: there is an accommodation strategy equilibrium where $M 1$ and $M 2$ can extract all the rent from their relationship with $R$ in $t=1$ by setting $w_{1}^{1 A}+w_{2}^{1 A}=$ $2+2 \delta^{r}(1-c)$. With such a strategy, manufacturers clearly increase their bargaining power towards the retailer compared to the one-shot game outcome. However, another condition is required to insure the existence of accommodation equilibria:

Corollary 1 The range of $\delta^{m}$ in which accommodation equilibria arise is non-empty only if $\gamma>\frac{4(1-c)}{4-5 c}$ for $c<\frac{4}{5}$

Proof. The above condition just states that $\gamma \widetilde{\delta} \leq 1$.

Retailer's and manufacturers' financial constraints (positive profit on both periods) have a key role to explain why accommodation equilibria can hold. Notice first that retailer's financial constraint define an upper bound for manufacturers' rents extraction which is an important condition of existence for the accommodation equilibrium. Without the retailer's financial constraint, each manufacturer would always have an incentive to overbid his rival's offer in the accommodation strategy. Second, from Corollary 1 accommodation equilibria exist only if retailer's discount factor is sufficiently greater than the manufacturers' discount factor. ${ }^{4}$ Indeed, if all firms have the same discount factor, from an accommodation situation where manufacturers saturate retailer's financial constraint in $t=1$, each firm would have an incentive to underbid his rival's offer in order to obtain the exclusivity. The insight is as follows. Assume that $\delta^{r}=\delta^{m}$ (i.e $\gamma=1$ ), when the discount factor increases, the expected gain from the accommodation strategy relatively to the exclusion strategy for manufacturers rises in $t=1$ but the future loss from this accommodation strategy also rises in $t=2$. At the end, a rise in $\delta$ gives no advantage to the accommodation strategy towards the exclusion strategy, and there is no accommodation Nash equilibrium in pure strategy. If now $\delta^{r}=\gamma \delta^{m}$, an increase in $\gamma$

\footnotetext{
${ }^{4}$ Note that this condition is not required in the linear demand case developed in extension.
} 
improves the relative profitability of the accommodation strategy in $t=1$ without changing his relative expected loss in $t=2$ since only the first period profits depend on $\delta^{r}$. Thus, if $\gamma$ is strong enough, manufacturers do not have any incentive to underbid his rival offer and accommodation equilibria in pure strategy are sustainable. Without the above two conditions, there would not therefore exist any pure Nash equilibrium but only mixed strategy equilibria would arise, like in Biglaiser and Vettas (2004).

Finally, our main conclusions are that:

(1) If producers' dependency towards a retailer is such that they can credibly threaten the latter to exit the market in case of a temporary breach in their relationships, this threat can be a source of bargaining power for manufacturers;

(2) Such a source of bargaining power is strong enough to enable manufacturers to annihilate entirely upstream competition.

An interesting parallel can be made with the results of Lewis and Yildirim (2002) in a learning-by-doing framework where no supply means a higher marginal cost for future periods (and not exit of the market). They qualitatively find the same result: a sole buyer maintains an inefficient supplier for future competition by splitting orders (mixed strategy equilibria). They do not however find that the manufacturers exploit the buyer's dependence through high wholesale transfers. Their result relies on the information asymmetry assumption. Because each manufacturer ignores the marginal cost of his rival, he cannot anticipate how much the retailer is willing to pay in order to preserve future competition. When costs are known, exclusive supply is much more likely.

Note that even if $c=0$, no accommodation equilibrium exists if $\delta^{m}<\frac{1}{2}$ just as no collusion with classical trigger strategies would be sustainable for $\delta^{r}<\frac{1}{2}{ }^{5}$

\footnotetext{
${ }^{5}$ Moreover, if collusive equilibria arise only in infinitely repeated games, the monopoly profit is reached here
} 


\section{Discussion on Assumptions}

This section briefly discusses the implications of our main assumptions of the model $(A 1-A 3)$ as well as the linear tariff contract the and the unit demand hypothesis.

\subsection{Introducing a probability of exit (or entry)}

A1 can be partially relaxed introducing a probability of exit for a manufacturer who has no order in $t=1$. Let denote this probability $\alpha$. Analytical expression remain the same unless $\delta^{i}$ is replaced by $\alpha \delta^{i}$, as exposed in the Appendix. Indeed, probability of presence for upstream competition only affects profits for the future period, and thus plays the same role than the discount factor. As $\alpha$ is reduced, the accommodation equilibria is less likely to happen given $\delta^{m}$, while the predation equilibrium is sustainable for a wider interval of $\delta^{m}$.

$A 3$ can be partially relaxed introducing a probability $\beta$ of entry for a new inefficient producer in $t=2$. The two-period game equilibria are modified exactly as when we introduce a probability of exit $\alpha$ with $\alpha=(1-\beta)$. On the contrary, the higher the probability of entry in $t=2$, the narrower (resp.wider) the interval of $\delta^{m}$ which sustains an accommodation equilibria (resp. predation equilibrium).

\subsection{Two-part tariff Contracts and Linear Demand}

We now examine the robustness of our results to the introduction of two-part tariff: wholesale prices may impact on quantities whereas fix part may be a tool of profit redistribution between upstream firms and the retailer. In the unit demand framework, the wholesale price plays in fact the role of unit transfer since the retailer may buy one unit to each manufacturer. The wholesale price was thus just a tool for sharing profit and it did not impact so much on quantity ordered.

for some high enough discount factor values, even if the number of periods is finite or known. 
In order to fully encompass quantities effect in firms' strategies, we introduce simple linear demand framework $Q=1-p$, where we assume that each manufacturer needs a minimum quantity of orders, $q_{i}^{1} \geq m$ in $t=1$ to remain active in $t=2$ (with $m<\frac{1}{4}$ ). Demand becomes now elastic and it is necessary to disentangle efficiency from profit sharing. However, note that because $M 2$ has to sell a minimum quantity $m$ (inelastic demand), wholesale price and transfer will be interrelated for this firm.

The following proposition shows that our main result still holds :

Proposition 2 (i) If $\delta^{r} \geq \frac{4 \mathrm{~cm}}{(1-c)^{2}}$, there are accommodation equilibria defined by: $w_{1}^{1 A}=0 ; T_{1}^{1 A}=$ $\frac{\delta^{r}(1-c)^{2}}{4}-w_{2}^{1 A} m+\frac{\left(1-w_{2}^{1 A}\right) w_{2}^{1 A}}{2}$ and $w_{2}^{1 A} \in\left[0 ; \frac{\delta^{r}(1-c)^{2}}{4 m}\right] ; T_{2}^{1 A}=\frac{\delta^{r}(1-c)^{2}}{4}-w_{2}^{1 A} m . R$ supplies from the two suppliers in $t=1$ and $w_{1}^{2 A}=0, T_{1}^{2 A}=\frac{1}{4}-\frac{(1-c)^{2}}{4}$ and $w_{2}^{2 A}=c, T_{2}^{2 A}=0$.

(ii) When $\delta^{r}<\frac{4 c m}{(1-c)^{2}}$, there is an exclusivity equilibrium where $w_{1}^{1 E}=0 ; T_{1}^{1 E}=\frac{c}{4}$ and $w_{2}^{1 E}=$ $0 ; T_{2}^{1 E}=\frac{1}{4} . R$ buys only from $M 1$ in $t=1$, and $w_{1}^{2 E}=0 ; T_{1}^{2 E}=\frac{1}{4}$.

Proof. See Appendix.

With two-part tariffs, as long as the variable part is positive or null, each manufacturer is in fact obliged to leave a rent to the retailer in order for the accommodation equilibrium to hold (disagreement pay-off). However, the retailer makes less profits than in a one-shot game repeated twice. If the retailer's discount factor is sufficiently low, there exists an exclusivity agreement where the most efficient manufacturer (M1) supplies the retailer for both periods. Note here that, contrary to the unit demand case, the discount factor do not have to be different to insure that accommodation equilibria are sustainable. It is indeed the retailer's discount factor, $\delta^{r}$, that is relevant here because of retailer's financial constraint. Since underbidding on wholesale price for getting exclusivity is not feasible for manufacturers (this would induce in fact negative wholesale prices), the difference in the discount factors between manufacturers and the retailer is no more a necessary condition for the existence of the accommodation strategy. ${ }^{6}$

\footnotetext{
${ }^{6}$ Note that with linear tariff contract, an accommodation strategy (pure Nash strategy) where wholesale
} 


\section{Conclusion}

This article shows in a simple two-period game that, if manufacturers are likely to exit the market in case of insufficient orders in the first period, they may exploit this threat of exit to capture the whole first period industry profit. Indeed, the retailer will accept to pay the high price to the manufacturers in order to secure upstream competition in the second period. The threat of exit appears here as a source of bargaining power for producers. Such a strategy is however prejudicial for the social welfare since an inefficient supplier is artificially maintained on the market for the sole reason of providing the retailer positive future profits. These results are still valid with various assumptions on demand and contracts.

prices are set to leave no intertemporal rent to the retailer is never an equilibrium because a slight wholesale price decrease from the most efficient seller is enough to convince the retailer to buy all to him, generating a positive profit that results from the slight margin made on each unit. Remember that otherwise the retailer would make no intertemporal profit on both periods. See the Appendix. 


\section{REFERENCES}

Allain M-L. (2002) "The Balance of Power Between Producers and Retailers : a Differentiation Model", Louvain Economic Review, 68 (3), 359-370.

Biglaiser G. and De Graba P. (2001) "Downstream Integration by a Bottleneck Input Supplier whose Regulated Wholesale Prices are Above Costs", The RAND Journal of Economics, $32,302-315$.

Biglaiser G. and Vettas N. (2004) "Dynamic Price Competition with Capacity Constraints and Strategic Buyers", CEPR Discussion Papers, 4315.

Bolton P. and Scharfstein D.(1990) "A Theory of Predation Based on Agency Problems in Financial Contracting", American Economic Review, 80(1): 93-106.

Chambolle C. and Villas-Boas S. (2007) "Buyer Power through Producer's Differentiation", Department of Agricultural \& Resource Economics, UCB, CUDARE Working Paper 1042;

Dobson P and Waterson P. (1999), "Retailer power: recent developments and policy implications", Economic Policy, April1999, p. 135 - 162.

Dobson P and Waterson P. (1997), "Countervailing power and consumer prices", The Economic Journal, 107, $418-430$.

Holström B. and Tirole J. (1997), "Financial intermediation, loanable funds and the real sector", Quarterly Journal of Economics, Vol. 62, p. 663-691.

Inderst R. and Mazzarotto N. (2006), "Buyer Power in Distribution", Manuscript prepared for the ABA Antitrust Section Handbook, Issues in Competition Law and Policy (W.D. Collins, (in preparation).

Inderst, R.and G.Shaffer (2006), "Retail Mergers, Buyer Power and Product Variety", The Economic Journal, Vol. 117, p. $45-67$.

Inderst R. and Wey C. (2007), "Buyer Power and Supplier Incentives", European Economic Review, Vol. 51, p. $647-667$. 
Lewis T.R and Yildirim H. (2002), "Managing Dynamic Competition", The American Economic Review, 92, 4, $779-797$. 


\section{APPENDIX}

\section{Proof of Proposition 1}

- $w_{1}^{1 A}+w_{2}^{1 A}=2+2 \delta^{r}(1-c)$ is an equilibrium iff:

- M1 has no incentive to deviate: His profit with this accommodation strategy is higher than the profit he would obtain in excluding $M 2$, given $w_{2}^{1 A}$ iff:

$$
\begin{gathered}
\left(2-w_{2}^{1 A}+2 \delta^{r}(1-c)\right)+2 \delta^{m} c>2\left(w_{2}^{1 A}-2 \delta^{r}(1-c)\right)+2 \delta^{m} \\
\Leftrightarrow w_{2}^{1 A}<\frac{2}{3}\left(1+(3 \gamma-1) \delta^{m}(1-c)\right)
\end{gathered}
$$

- M2 has no incentive to deviate: His profit with this accommodation strategy is higher than the profit he would obtain in excluding $M 1$, given $w_{1}^{1 A}$ iff:

$$
\begin{gathered}
2-w_{1}^{1 A}+2 \delta^{r}(1-c)-c>2\left(w_{1}^{1 A}-2 \delta^{r}(1-c)-c\right)+2 \delta^{m}(1-c) \\
\Leftrightarrow w_{1}^{1 A}<\frac{2}{3}\left(1+\frac{c}{2}+(3 \gamma-1) \delta^{m}(1-c)\right)
\end{gathered}
$$

(2) is rewritten with $w_{1}^{1 A}=2+2 \delta^{r}(1-c)-w_{2}^{1 A}$, leading to $\left(2^{\prime}\right)$ :

$$
w_{2}^{1 A}>\frac{1}{3}\left(4+2 \delta^{m}(1-c)-c\right)
$$

There is a continuum of equilibria such that $w_{1}^{1 A}+w_{2}^{1 A}=2+2 \delta^{r}(1-c)$, if $w_{2}^{1 A} \in$ $\left[\frac{1}{3}\left(4+2 \delta^{m}(1-c)-c\right), \frac{2}{3}\left(1+(3 \gamma-1) \delta^{m}(1-c)\right)\right]$. This interval is non empty if and only if $\delta^{m}>\widetilde{\delta}=\frac{2-c}{2(1-c)(3 \gamma-2)}$.

- $w_{1}^{1 P}=w_{2}^{1}-2 \delta^{r}(1-c)$ and $w_{2}^{1 P}=c$ is an exclusion equilibrium iff:

- M2 has no incentive to raise his price, which is immediate. 
- The resulting profit for $M 1$ is higher with a exclusion strategy than with an accommodation one:

$$
2\left(w_{2}^{1}-2 \delta^{r}(1-c)\right)+2 \delta^{m}>w_{2}^{1}+2 \delta^{r}(1-c)+2 \delta^{m} c \Leftrightarrow w_{2}^{1}>(6 \gamma-2) \delta^{m}(1-c)
$$

As $w_{2}^{1}>c$, an exclusion equilibrium exist iff $\Leftrightarrow \delta^{m}<\frac{c}{2(3 \gamma-1)(1-c)}=\delta^{*}$.

\section{Proof of Corollary 1}

For accommodation equilibria to exist, we need to satisfy $\delta^{r}=\gamma \widetilde{\delta}<1$ which translates into $\gamma>\frac{4(1-c)}{4-5 c}$. When $c>\frac{4}{5}$ this condition is not binding since $\gamma>1>\frac{4(1-c)}{4-5 c}$.

\section{Proof of Extensions}

\section{A1 and A3}

There is a continuum of accommodation equilibria defined by the following conditions: $w_{2}^{1 A} \in$ $\left[\frac{1}{3}\left(4+2 \delta^{m} \alpha(1-c)-c\right), \frac{2}{3}\left(1+\alpha \delta^{m}(1-c)(3 \gamma-1)\right)\right]$ and $w_{1}^{1 A}+w_{2}^{1 A}=2+2 \alpha \delta^{r}(1-c)$ when

$\delta^{m} \in\left[\frac{2-c}{2 \alpha(1-c)(3 \gamma-2)}, 1\right]$. There is one exclusion equilibrium, where $w_{1}^{1 E}=c-2 \delta^{r} \alpha(1-c)$, $w_{2}^{1 E}=c$ when $\delta^{m} \in\left[0, \frac{c}{2 \alpha(1-c)(3 \gamma-1)}\right]$.

\section{Proof of Proposition 2}

In this section, demand is linear and contracts between manufacturers and the retailer are two-part tariffs. We also assume that each manufacturer needs a minimum quantity ordered to survive $\left(q_{1}>m\right.$ and $q_{2}>m$ with $\left.m<\frac{1}{4}\right)$. We solve the game backward.

- Second period

- If $M 1$ is a monopoly, he offers a contract $w_{1}^{2}=0, T_{1}^{2}=\frac{1}{4}$ to $R$.

Discounted payoffs are: $\pi_{M 1}=\frac{\delta^{m}}{4}, \pi_{M 2}=0, \pi_{R}=0$. 
- If $M 2$ is a monopoly, he offers a contract $w_{2}^{2}=c, T_{1}^{2}=\frac{(1-c)^{2}}{4}$ to $R$.

Discounted payoffs are: $\pi_{M 1}=0, \pi_{M 2}=\frac{\delta^{m}(1-c)^{2}}{4}, \pi_{R}=0$.

- If $M 1$ and $M 2$ compete, $M 1$ offers a contract $w_{1}^{2}=0, T_{1}^{2}=\frac{1}{4}-\frac{(1-c)^{2}}{4}$ and $M 2$ offers a contract $w_{2}^{2}=c, T_{2}^{2}=0$ to $R$. Discounted payoffs are: $\pi_{M 1}=\delta^{m}\left(\frac{1}{4}-\frac{(1-c)^{2}}{4}\right), \pi_{M 2}=$ $0, \pi_{R}=\frac{\delta^{r}(1-c)^{2}}{4}$.

\section{- First period}

We propose one candidate for the Nash equilibrium of 'accommodation' and we check ex-post that there is no profitable deviation.

\section{- Accommodation}

Let first assume $\left\{w_{1}^{1 A}=0 ; T_{1}^{1 A}\right.$ given $\}$. The maximum fixed fee $M 2$ can set in order to induce an accommodation strategy is such that retailer's profit is higher if supplied by the two manufacturers rather than just $M 1$ :

$$
\left(p-w_{1}^{1 A}\right)(1-p-m)+\left(p-w_{2}^{1 A}\right) m-T_{1}^{1 A}-T_{2}^{1 A}+\frac{\delta^{r}(1-c)^{2}}{4}>\frac{1}{4}-T_{1}^{1 A} \Leftrightarrow T_{2}^{1 A} \leq \frac{\delta^{r}(1-c)^{2}}{4}-w_{2}^{1 A} m
$$

M2's profit are therefore:

$$
\pi_{M 2}^{A}=\left(w_{2}^{1 A}-c\right) m+T_{2}^{1 A}=\frac{\delta^{r}(1-c)^{2}}{4}-m c
$$

The candidate equilibrium values for $M 2$ are thus:

$$
w_{2}^{1 A} \in\left[0 ; \frac{\delta^{r}(1-c)^{2}}{4 m}\right] \quad ; \quad T_{2}^{1 A}=\frac{\delta^{r}(1-c)^{2}}{4}-w_{2}^{1 A} m>0
$$

Let assume $\left\{T_{2}^{1 A}=\frac{\delta^{r}(1-c)^{2}}{4}-w_{2}^{1 A} m ; w_{2}^{1 A}\right.$ given; $\left.w_{1}^{1 A}=0\right\}$, the maximum fixed fee $M 1$ can set in order to induce an accommodation strategy is such that retailer's profit is higher if supplied by the two manufacturers rather than just $M 2$ :

$p(1-p-m)+\left(p-w_{2}^{1 A}\right) m-T_{1}^{1 A}-T_{2}^{1 A}+\frac{\delta^{r}(1-c)^{2}}{4}>\left(\frac{1+w_{2}^{1 A}}{2}-w_{2}^{1 A}\right)\left(1-\frac{1+w_{2}^{1 A}}{2}\right)-T_{2}^{1 A}$ 


$$
\Leftrightarrow T_{1}^{1 A} \leq \frac{\delta^{r}(1-c)^{2}}{4}-w_{2}^{1 A} m+\frac{\left(1-w_{2}^{1 A}\right) w_{2}^{1 A}}{2}
$$

M1's profit are therefore: $\pi_{M 1}^{A}=T_{1}^{1 A}+\delta^{m}\left(\frac{1}{4}-\frac{(1-c)^{2}}{4}\right)$.

The candidate equilibrium values for $M 1$ are thus:

$$
w_{1}^{1 A}=0 \quad ; \quad T_{1}^{1 A}=\frac{\delta^{r}(1-c)^{2}}{4}-w_{2}^{1 A} m+\frac{\left(1-w_{2}^{1 A}\right) w_{2}^{1 A}}{2}
$$

We check first that there are no incentives to deviate for either $M 1$ or $M 2$ towards a different unit price.

- Does $M 1$ would have an incentive to set a price $w_{1}^{1 A}>0$ ?

$\pi_{M 1}^{A}=w_{1}^{1 A} \frac{\left(1-2 m-w_{1}^{1 A}\right)}{2}+\left(\frac{\left(1-w_{1}^{1 A}\right)^{2}-(1-c)^{2}}{4}+\left(w_{1}^{1 A}-c\right) m+\delta^{r} \frac{(1-c)^{2}}{4}\right)+\delta^{m}\left(\frac{1}{4}-\frac{(1-c)^{2}}{4}\right)$ $\frac{\partial \pi_{M 1}^{A}}{\partial w_{1}^{1 A}}=\frac{-w_{1}^{1 A}}{2}<0$. There is no incentive to deviate for $M 1$.

- Does $M 2$ would have an incentive to set a price $w_{2}^{1 A} \neq c$ ?

$\pi_{M 2}^{A}=\left(w_{2}^{1 A}-c\right) m-w_{2}^{1 A} m+\delta^{r} \frac{(1-c)^{2}}{4}$

$\frac{\partial \pi_{M 2}^{A}}{\partial w_{2}^{1 A}}=0$. There is no incentive to deviate.

- Does $M 2$ would have an incentive to set a price $w_{2}^{1}=0$ ?

Retailer goes for accommodation as long as $\frac{1}{4}-T_{1}^{1 A}-T_{2}^{1 A}+\delta^{r} \frac{(1-c)^{2}}{4} \geq \frac{1}{4}-T_{1}^{1 A}$ $\Leftrightarrow T_{2}^{1 A} \leq \delta^{r} \frac{(1-c)^{2}}{4}$

With $w_{2}^{1 A}=0, \pi_{M 2}^{A}=(0-c) \frac{1}{4}+\delta^{r} \frac{(1-c)^{2}}{4}$ while if $w_{2}^{1 A}=c, \pi_{M 2}^{A}=-c m+\delta^{r} \frac{(1-c)^{2}}{4}$. Since $m<\frac{1}{4}, M 2$ has no incentive to deviate.

Finally, we have to check that each manufacturer does not have an incentive to deviate towards an 'exclusion' strategy. To exclude his rival, a manufacturer has to set a lower price than his rival such that the retailer may find it more profitable to sign for exclusivity. Manufacturer $M 1$ gets the exclusivity if the price $w_{1}^{1 E}$ is such that:

$\left(p-w_{1}^{1 E}\right)(1-p-m)+\left(p-w_{2}^{1 E}\right) m-T_{1}^{1 E}-T_{2}^{1 A}+\delta^{r} \frac{(1-c)^{2}}{4}<\left(p-w_{1}^{1 E}\right)(1-p)-T_{1}^{1 E}$ 
This leads to $w_{1}^{1 E}<w_{2}^{1 A}+\frac{1}{m}\left(T_{2}^{1 A}-\delta^{r} \frac{(1-c)^{2}}{4}\right) \Longleftrightarrow w_{1}^{1 E}<0$. This is not possible.

Manufacturer $M 2$ gets the exclusivity if the price $w_{2}^{1 E}<0$ which is not possible.

None of the manufacturers has therefore an incentive to deviate. The equilibrium found has however to provide positive profits to each agent. Payoffs are:

$$
\begin{aligned}
& \pi_{R}^{A}=\frac{\left(1-w_{2}^{1 A}\right)^{2}}{4}-\delta^{r} \frac{(1-c)^{2}}{4}+w_{2}^{1 A} m \\
& \pi_{M 1}^{A}=\frac{\left(1-w_{2}^{1 A}\right)^{2}}{4}-\delta^{r} \frac{(1-c)^{2}}{4}+w_{2}^{1 A} m+\delta^{m}\left(\frac{1}{4}-\frac{(1-c)^{2}}{4}\right) \\
& \pi_{M 2}^{A}=\delta^{r} \frac{(1-c)^{2}}{4}-c m
\end{aligned}
$$

In order to insure that $\pi_{M 2}^{A}>0$, the following condition has to hold: $\delta^{r}>\frac{4 c m}{(1-c)^{2}}$.

- Exclusivity

When $\delta^{r}<\frac{4 c m}{(1-c)^{2}}, M 2$ cannot prevent $M 1$ from excluding him. Thus there is only an exclusion equilibrium where $M 1$ sets $w_{1}^{1 E}=0$ and $T_{1}^{1 E}=\frac{c}{4}-\varepsilon ; w_{2}^{1 E}=0$ and $T_{2}^{1 E}=\frac{1}{4}$ leading $M 1$ to get the exclusivity.

\section{Additional Case: Linear Demand and Contracts}

Let assume $c<\frac{1}{2}, m<\frac{1-c}{6}$ and linear wholesale prices contracts between $\{M 1, M 2\}$ and $R$, in $t=2$, the final retail price is $p^{2}=\frac{1+w_{i}^{2}}{2}$ with $i=\{1,2\}$.

- Second period If only $M 1$ (resp. $M 2)$ is active, his profit is $\pi_{M 1}^{2}=\delta^{m} \frac{1}{8}\left(\operatorname{resp} . \pi_{M 2}^{2}=\right.$ $\delta^{m} \frac{(1-c)^{2}}{8}$ ) and R's profit is $\pi_{R}^{2}=\delta^{r} \frac{1}{16}$ (resp. $\left.\pi_{R}^{2}=\delta^{r} \frac{(1-c)^{2}}{16}\right)$.

If manufacturers compete, $M 2$ has no profit and $M 1$ gets $\pi_{M 1}^{2}=\delta^{m} \frac{c(1-c)}{2}, R$ gets $\pi_{R}^{2}=\delta^{r} \frac{(1-c)^{2}}{4}$ and $q_{M 1}^{2}=\frac{1-c}{2}$.

- First period There are two possible accommodation strategies for $M 2$ given $w_{1}^{1}$ :

(1) $M 2$ sets a strictly lower wholesale price than $M 1: w_{1}^{2}<w_{1}^{1}$. In such a case, the retailer will buy the minimum order $m$ to the most expensive supplier $M 1$. The residual demand addressed to $M 2$ is therefore $q_{M 2}^{1}=1-p-m$. 
The retailer maximizes $\pi_{R}^{1}=\left(p-w_{1}^{1}\right) m+\left(p-w_{2}^{1}\right)(1-p-m)$ leading to $p^{1}=\frac{1+w_{2}^{1}}{2}$. Therefore, $M 2$ best response is given by $w_{2}^{1}\left(w_{1}^{1}\right)=\frac{1+c}{2}-m$, i.e $w_{2}^{1}$ such that $\pi_{R}^{1}\left(w_{2}^{1}\right)=0$.

(2) $M 2$ sets a strictly higher wholesale price than $M 1: w_{1}^{2}>w_{1}^{1}$. In such a case, the retailer will buy him the minimum order $m$. The demand addressed to $M 2$ is therefore $q_{M 2}^{1}=m$

The retailer maximizes $\pi_{R}^{1}=\left(p-w_{1}^{1}\right)(1-p-m)+\left(p-w_{2}^{1}\right) m$ leading to $p^{1}=\frac{1+w_{1}^{1}}{2}$. Therefore, $M 2$ best response is given by $w_{2}^{1}\left(w_{1}^{1}\right)=\frac{1+w_{1}^{1}\left(w_{1}^{1}+4 m-2\right)}{4 m}$, such that $M 2$ maximization his profits.

The same reasoning apply to find $M 1$ best response, leading to $w_{1}^{1}\left(w_{2}^{1}\right)=\frac{1}{2}-m$ when $w_{1}^{1}<w_{2}^{1}$ and $w_{1}^{1}\left(w_{2}^{1}\right)=\frac{1+w_{2}^{1}\left(w_{2}^{1}+4 m-2\right)}{4 m}$ when $w_{1}^{1}>w_{2}^{1}$.

There are two Nash equilibria "candidates" for accommodation:
$(\mathrm{A}): w_{2}^{1 A}=\frac{1-2 m+c}{2}$ and $w_{1}^{1 A}=\frac{1-2 m+c}{2}+\frac{(1+2 m-c)^{2}}{16 m}$ where $w_{1}^{1 A}>w_{2}^{1 A}$ and
(B): $w_{1}^{1 A}=\frac{1-2 m}{2}$ and $w_{2}^{1 A}=\frac{1-2 m}{2}+\frac{(1+2 m)^{2}}{16 m}$ where $w_{2}^{1 A}>w_{1}^{1 A}$.

However, a slight decrease in the wholesale price (for instance $\widetilde{w}_{1}^{1}=\frac{1-2 m}{2}-\epsilon$ or $\widetilde{w}_{2}^{1}=$ $\frac{1-2 m+c}{2}-\epsilon$ ) is enough to convince the retailer to get exclusive supply from $M 1$ (or $M 2$ ) because then not only $\widetilde{\pi}_{R}>0$ but also the manufacturer makes an additional profit on each unit sold. 